\title{
Modelling Interdependent Cascading Failures in Real World Complex Networks using a Functional Dependency Model
}

\author{
Sarah Dunn, Matthew Holmes and Sean Wilkinson \\ School of Civil Engineering and Geosciences, Newcastle University, UK
}

\begin{abstract}
Infrastructure systems are becoming increasingly complex and interdependent. As a result our ability to predict the likelihood of large-scale failure of these systems has significantly diminished and the consequence of this is that we now have a greatly increased risk of devastating impacts to society.

Traditionally these systems have been analysed using physically-based models. However, this approach can only provide information for a specific network and is limited by the number of scenarios that can be tested. In an attempt to overcome this shortcoming, many studies have used network graph theory to provide an alternative analysis approach. This approach has tended to consider infrastructure systems in isolation, but has recently considered the analysis of interdependent networks through combination with percolation theory. However, these studies have focused on the analysis of synthetic networks and tend to only consider the topology of the system.

In this paper we develop a new analysis approach, based upon network theory, but accounting for the hierarchical structure and functional dependency observed in real world infrastructure networks. We apply this method to two real world networks, to show that it can be used to quantify the impact that failures within an electricity network have upon a dependent water network.
\end{abstract}

Keywords: Resilience, Interdependency, Network Graph Theory, Hazard

\section{MODELLING INTERDEPENDENT INFRASTRUCTURE SYSTEMS}

Network theory is an area of graph theory that concerns the study of graphs, which are mathematical structures used to model relationships between discrete objects. In this context a 'graph', or network, consists of nodes and connecting edges. The study of networks is a relatively young area of research and has been largely driven by the desire to study real world networks, such as social and biological networks. One of the main contributions of this area of research is the discovery and classification of underlying patterns in many real world networks. There are four main classes of network, into which the many real world networks (including infrastructure systems) can be placed. Each of these classes are distinguished by a degree distribution, where the degree of a node is the number of connections it has with other nodes and the degree distribution of a network is the probability distribution of these degrees for the whole network.

The first documented network class was the random graph ${ }^{1}$, which has since been shown to be a poor

\footnotetext{
1 Erdos, P. \& Renyi, A. On The Evolution of Random Graphs. Publication of the Mathematical Institute of the Hungarian Academy of Sciences 5, 17-61 (1960).
} 
representation of real world networks. However, this network class formed the basis for the small-world network class, developed by Watts and Strogatz ${ }^{2}$, which has been shown to replicate a range of real world networks including subway systems ${ }^{3}$. These two classes of network are characterised by a Poisson degree distribution; however, Barabasi and Albert discovered that real world networks tend to form a power law degree distribution ${ }^{4}$. Networks that follow this power law are more commonly known as scale-free networks and include the Internet and the WorldWide-Web. ${ }^{5}$ Other real world networks, including power grids, have been found to have an exponential degree distribution and are termed 'exponential networks'. 6,7,8

The main advantage of classifying a real world network into a network class is that it gives an insight into the inherent hazard tolerance of a network. For example, infrastructure systems have been shown to fall into either the scale-free or exponential network class ${ }^{9,10}$ and these networks consist of a small number of highly connected nodes and a large number of weakly connected nodes. As such they have been shown to be vulnerable to targeted attack, as this will tend to remove one of the highly connected nodes to cause the maximum disruption, and also resilient to random hazard, as this will tend to remove one of the many weakly connected components ${ }^{11}$.

In this paper, we are considering the hazard tolerance of two real world networks and whether we can use this approach to gain an insight into their resilience to different attack strategies. We have constructed a network model of both networks using data obtained from a real electricity distribution network and a real water network that is, in reality, connected to the electricity network. The electricity network consists of 883 nodes (representing the Grid Supply Points, Bulk Supply Points, Primary Substations and Distribution Substations) and 3039 connecting links; whilst the water network consists of 144 nodes (representing the Source Nodes, Pumping Stations, Water Treatment Works, Service Reservoirs and Demand Nodes) and 305 connecting links. For a detailed explanation of the process used to model real world networks using network graph theory, the reader is directed to Dunn et al. ${ }^{12}$ The degree distributions for these two networks have been shown in Figure 1. From this figure, it can be seen that the water network clearly follows an exponential distribution (forming a straight line when the results are plotted on a log-linear axis). However, it is more difficult to classify the electricity network, as it does not appear to fit exactly into one network class. This is due to the presence of a large number of small degree nodes. As the water network can be classed as exponential, it should be resilient to random hazard and vulnerable to targeted attack. However, classifying these networks does not necessarily give an insight into the hazard tolerance of the water network when the electricity network is disrupted, as the dependent links between these networks are not considered. To establish this relationship, an additional analysis approach is needed.

2 Watts, D. J. \& Strogatz, S. H. Collective dynamics of 'small-world' networks. Nature 393, 440-442 (1998).

3 Latora, V. \& Marchiori, M. Is the Boston subway a small-world network? Physica a-Statistical Mechanics and Its Applications 314, 109113 (2002).

4 Barabasi, A. L. \& Albert, R. Emergence of scaling in random networks. Science 286, 509-512 (1999).

5 Barabasi, A. L., Albert, R. \& Jeong, H. Scale-free characteristics of random networks: the topology of the World-Wide Web. Physica A 281, 69-77 (2000).

6 Amaral, L. A. N., Scala, A., Barthelemy, M. \& Stanley, H. E. Classes of small-world networks. Proceedings of the National Academy of Sciences of the United States of America 97, 11149-11152 (2000).

7 Liu, J. Z. \& Tang, Y. F. An exponential distribution network. Chinese Physics 14, 643-645 (2005).

8 Wilkinson, S. M. \& Henderson, N. A. in 14th World Conference on Earthquake Engineering (Beijing, China, 2008).

9 Crucitti, P., Latora, V. \& Marchiori, M. A topological analysis of the Italian electric power grid. Physica a-Statistical Mechanics and Its Applications 338, 92-97, doi:10.1016/j.physa.2004.02.029 (2004).

10 Wilkinson, S., Dunn, S. \& Ma, S. The vulnerability of the European air traffic network to spatial hazards. Natural Hazards 60, 1027-1036, doi:10.1007/s11069-011-9885-6 (2012).

11 Albert, R., Jeong, H. \& Barabasi, A. L. Error and Attack Tolerance of Complex Networks. Nature 406, 378-382 (2000).

12 Dunn, S., Fu, G., Wilkinson, S. \& Dawson, R. Network theory for infrastructure systems modelling. Proceedings of the ICE - Engineering Sustainability 166, 281-292 (2013). 

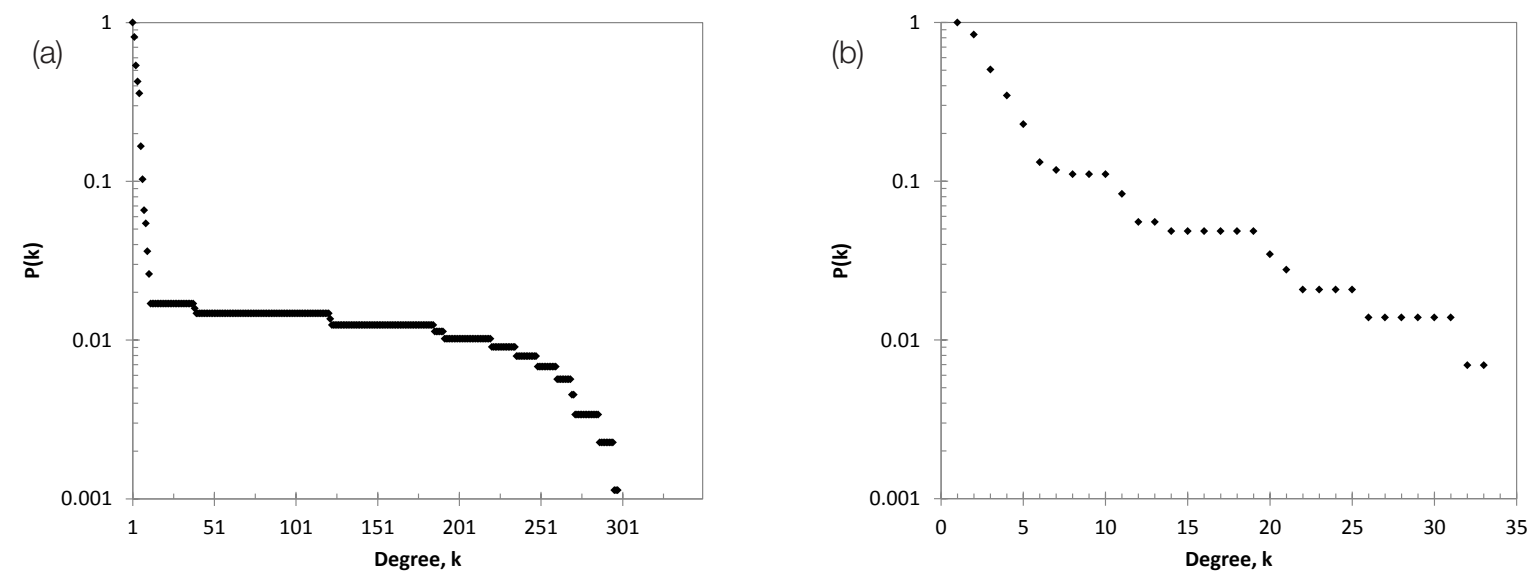

Figure 1: The degree distributions for the (a) energy and (b) water networks.

Previous studies have modelled the interdependency between two coupled networks by using additional links, which are usually directed, to represent the dependence of components in one network upon those in another. One study by Buldyrev et al. ${ }^{13}$ assessed impact of failure within Internet infrastructure as a result of disruption to electricity infrastructure. In their study, they identified the dependent links between the two networks by coupling each Internet server to the geographically nearest power station. They then removed power stations, at random, and observed the resulting impact to the Internet network, following an iterative process to fail connected nodes. Nodes were deemed to have failed if (1) all of their connected nodes were failed and/or (2) their dependent node in the other network was failed. They showed that these networks were extremely sensitive to random failures and that the removal of a small fraction of nodes in one network was sufficient to produce an iterative cascade of failures in the interdependent network.

Further studies have attempted to identify a critical threshold (or proportion of failed nodes) which induces this cascade of failures in the dependent network, by combining network theory with percolation theory. One notable study by Gao et al. ${ }^{14}$, developed this approach and used it to study the failures between two partially interdependent random networks. In their study, they 'fail' a proportion of nodes in one network and observe how the failure propagates to the connected network, by defining two conditions for failure: (1) nodes fail if they do not belong to the largest cluster of nodes, and (2) nodes also fail if they depend on the failed nodes in the other network.

However, this percolation theory approach only considers the topology of the network and does not consider the direction of flow in these networks or their hierarchical structure. For example, in electricity infrastructure a Primary Substation can only operate if it is connected to, at least one, Bulk Supply Point and this is not captured in a purely topological model. Therefore, we develop a new approach which recognises that nodes are used to represent a range of components and that flow between these components is not always bi-directional.

\section{DEVELOPMENT OF FUNCTIONAL DEPENDENCY MODEL}

We develop what we term a 'functional dependency' model which incorporates the hierarchical structure of real world infrastructure networks and the direction of flow through the use of directed links. Following traditional network theory models, we use nodes to represent the different components in both networks, however, unlike traditional models we record the type of component that the node represents. Figure 2 shows the type, and number, of each component in both the electricity (red) and water (blue) networks.

By recording the different types of nodes we can ensure that the hierarchical structure, observed in real world networks, is maintained (e.g. electricity can flow from a Grid Supply Point to a Bulk Supply Point, but not vice versa). We could simulate the flow of service in these networks using a flow model, such as that presented by Dunn and

13 Buldyrev, S. V., Parshani, R., Paul, G., Stanley, H. E. \& Havlin, S. Catastrophic cascade of failures in interdependent networks. Nature 464, 1025-1028, doi:10.1038/nature08932 (2010).

14 Gao, J. X., Buldyrev, S. V., Stanley, H. E. \& Havlin, S. Networks formed from interdependent networks. Nat. Phys. 8, 40-48 (2012). 
Wilkinson ${ }^{15}$; however, we deem a detailed study of flow and capacity outside the scope of this paper and therefore use the hierarchical structure to make an assumption regarding the capacity of each of the 'supply' components (e.g. Grid Supply Points and Service Reservoirs). The intention is not to replicate the extant network precisely, but rather to develop a plausible model that captures the essential failure characteristics of the real network.

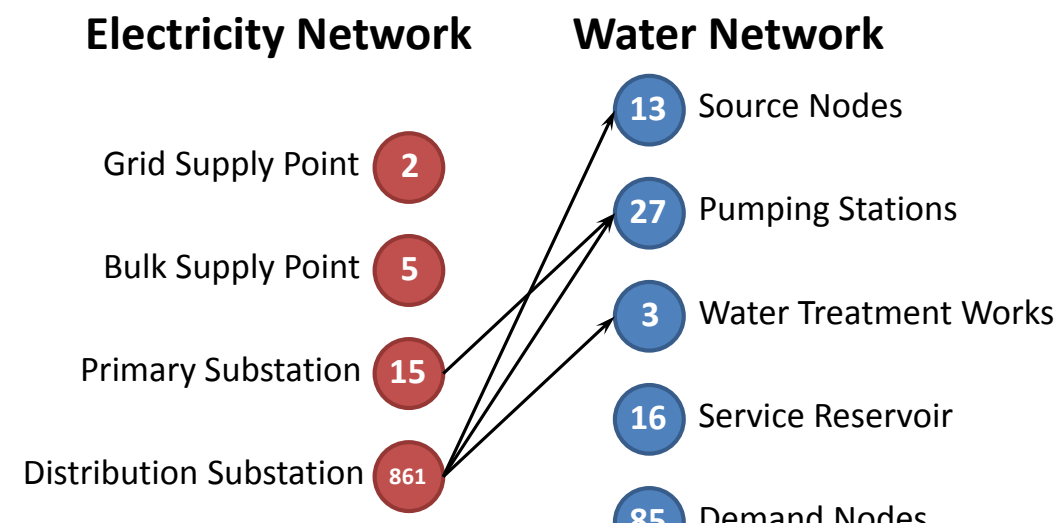

Figure 2: Showing the number of each individual component in the energy (red) and water (blue) networks and the interconnectivity of these components (black lines).

In the electricity network we achieve this by using Dijkstra's algorithm to calculate the length, in terms of the number of nodes traversed, of the shortest route from each Distribution Substation to each Primary Substation. It is assumed that the closest Primary Substation supplies the Distribution Substation under normal operational conditions.

Based on discussions with local experts, we also define the limit to the area a primary substation might supply in an emergency as 50\% greater than its supply boundary in its typical configuration.

In the water network, the dependencies of the different components in the raw water sections of the network are easily understood, they take the shape of dendritic patterns feeding into the three water treatment works which then feed directly into service reservoirs. The downstream network requires more interpretation. In addition to the connectivity of the network, the ability of a service reservoir to feed a demand node is based upon their respective elevations. If the reservoir level was more than 20 metres above the highest property in the demand node then it can be supplied (the 20 meter difference accounts for the need to deliver a minimum level of pressure to a consumer's property). It is assumed that pumping stations can provide a pressure equivalent to the level of the highest point they supply.

The identification of dependent links between the networks is simplified because, as major consumers, the water components generally have named substations. The proximal substation was used for the two components where this was not the case. Between these networks we identified 31 dependent links and the components they connect have been shown in Figure 2 (black arrows).

In a similar manner to previous studies, we randomly fail a proportion of nodes in the electricity network and observe how this failure propagates to the dependent water network, by defining two conditions of failure. Nodes in the water network are deemed to have failed if they are (1) no longer connected to at least one functional 'parent' node (e.g. a node that is directly above them in the hierarchical structure), or (2) are connected to a dependent node in the other network which has been failed. However, unlike previous studies, we observe how the failure of components at different levels in the electricity network impacts the water network. We initially fail a proportion of nodes of different components (termed primary failure) and observe how this failure cascades throughout the network (removing further nodes, termed secondary failure), before considering how this failure propagates to the water network. The results of this analysis are shown in Figure 3.

15 Dunn, S. \& Wilkinson, S. Identifying Critical Components in Infrastructure Networks Using Network Topology. Journal of Infrastructure Systems 19, 157-165, doi:10.1061/(ASCE)IS.1943-555X.0000120 (2013). 

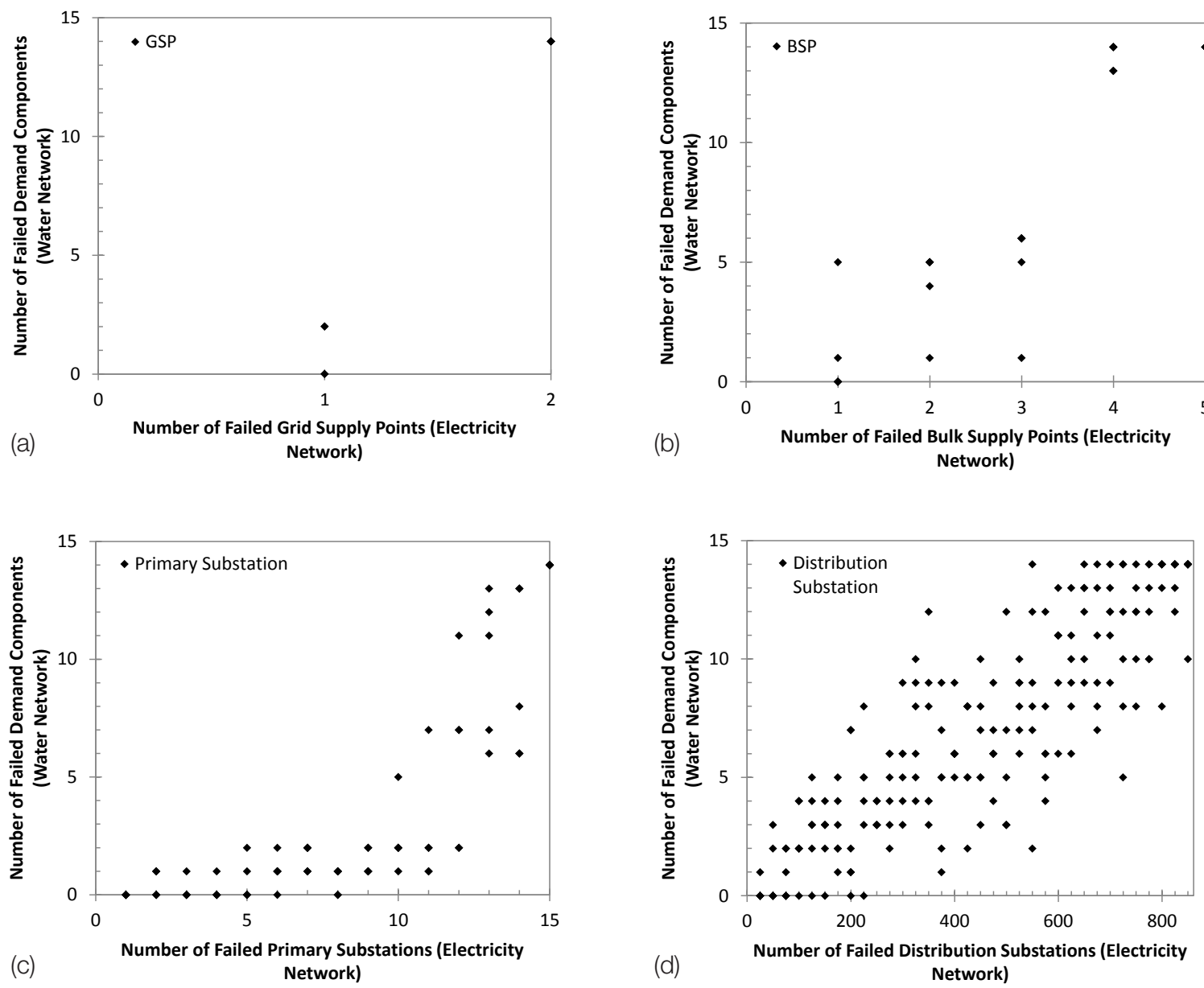

Figure 3: Showing the number of failed demand nodes in the water network, due to the failure of (a) the grid supply points, (b) bulk supply points, (c) primary substations and (d) distribution substations in the electricity network.

From Figure 3, it can be seen that the removal of different components in the electricity network have different impacts upon the water network. This is expected; removing a distribution substation is unlikely to affect other nodes but the effect of a lost bulk supply point will cascade through the system. However, it is interesting to note the different patterns of impacts caused by the failures at different levels in the hierarchy. For example, it can be seen that the correlation between primary substations failures and failed demand components is non-linear, and appears to increase exponentially (Figure 3(c)). By contrast the correlation between the number of failed distribution substations and the number of demand components (Figure $3(d)$ ) is linear, although there is a large amount of scatter in the results.

It is also evident that the complete failure of the electricity network (shown on the extreme right of the graphs in Figure 3) does not result in the complete failure of the water network. This is due to the presence of the service reservoirs (which do not require a supply of electricity to function) meaning that approximately $80 \%$ of the demand components network remain functional. It should be noted that we only perform a static analysis, and therefore do not capture the depletion of these resources; however, these components typically have sufficient capacity to last longer than the power companies' expected return to service time. 


\section{CONCLUSIONS}

In this paper we have presented a new approach to the analysis of interdependent failures between two coupled networks. Our approach is based upon traditional network theory, modelling the networks as a series of nodes and connecting links, but maintains the hierarchical structure of real world infrastructure networks and also makes an assumption of the supply capacity of each of the 'supply' nodes through the use of directed links. We have applied this approach to analyse two real world networks, an electricity network and a dependent water network, to assess how failures within the electricity network propagate to the water network. Through this analysis it was shown that the failure of different components in the electricity network can have vastly different impacts to the water network. This approach could be used in future studies to increase the validity of using network theory models to assess the impacts of cascading failures between two coupled networks.

\section{ACKNOWLEDGEMENTS}

Sarah Dunn is an EPSRC Doctoral Prize Fellow at Newcastle University and Matthew Holmes is a Stream Research student funded by EPSRC, United Utilities, Severn Trent Water and Yorkshire Water and their support is also gratefully acknowledged. 